\title{
Assessing health-related quality of life in patients with breast cancer: a reply to Maratia et al
}

\author{
Karin Ribi ${ }^{1}$ (1) Alan Coates $^{2} \cdot$ Lynette Blacher $^{3} \cdot$ Meredith M. Regan $^{4}$. \\ Richard D. Gelber ${ }^{5} \cdot$ Jürg Bernhard $^{6}$
}

Accepted: 24 August 2017/Published online: 5 September 2017

(C) Springer International Publishing AG 2017

In 2016, a publication on health-related quality of life (HRQoL) assessment in patients with breast cancer reported on a systematic and standardized comparison of available instruments using the evaluation measures of patient-reported-outcomes (EMPRO) tool [1]. In this review, eight HRQoL measures were compared including 32 studies reporting on psychometric properties or the validation process. One of the instruments included in this review is the International Breast Cancer Study Group (IBCSG) quality of life (QoL) Core Form [2]. Although we are pleased that the IBCSG QoL Core Form could be recommended for assessing health-related quality of life in breast cancer [1], the description of specific characteristics of the IBCSG form and the rating of this instrument based on EMPRO [3] requires clarification and further discussion of the development research overlooked during the rating.

\footnotetext{
Karin Ribi

karin.ribi@ibcsg.org

Alan Coates

alan.coates@ibcsg.org

Lynette Blacher

blacher.lynette@fstrf.org

Meredith M. Regan

mregan@jimmy.harvard.edu

Richard D. Gelber

gelber@jimmy.harvard.edu

Jürg Bernhard

juerg.bernhard@ibcsg.org

1 International Breast Cancer Study Group (IBCSG)

Coordinating Center, Effingerstrasse 40, 3008 Bern,

Switzerland

2 International Breast Cancer Study Group and University of Sydney, Sydney, Australia
}

Although Maratia et al. [1] conducted a predefined systematic search and selection of papers, they used only a summary paper that was published in 1997 to describe the IBCSG approach [2]. More detailed psychometric data for the IBCSG QoL Core Form have been published in many other studies as described in the following paragraphs.

The purpose for the development of the IBCSG QoL Core Form was to specifically assess the impact of adjuvant treatment on QoL in breast cancer patients participating in clinical trials in an international setting with multiple cultures and countries [2]. According to this specific purpose, the measure had to be applicable within clinical routine, taking into account the complex logistics of large-scale international trials involving different health care systems [4].

3 International Breast Cancer Study Group, Data Management Center, Amherst, NY, USA

4 International Breast Cancer Study Group Statistical Center, Dana-Farber Cancer Institute, Harvard Medical School, Boston, MA, USA

5 International Breast Cancer Study Group Statistical Center, Dana-Farber Cancer Institute, Harvard Medical School, Harvard T.H. Chan School of Public Health, Frontier Science and Technology Research Foundation, Boston, MA, USA

6 International Breast Cancer Study Group Coordinating Center and Bern University Hospital, Inselspital, Bern, Switzerland 
The IBCSG QoL Core Form is not restricted to one dimension as claimed by Maratia et al. [1]. The IBCSG Core Form consists of single-item linear analog selfassessment (LASA) indicators of components of QoL shown to be affected by breast cancer, surgery, chemotherapy, and endocrine therapy. These include global indicators for physical well-being [5, 6], mood [5-7], coping effort $[5,6,8]$, and perceived health status (utility) [9]. The specific indicators for side effects were nausea/ vomiting [2], appetite [5, 6], flushing [2], and arm restriction [2]. The only additional paper that was cited by Maratia et al. in reference to the IBCSG QoL Core Form, unfortunately used a summary score for their validation of the Portuguese version, which may led to the understanding that this questionnaire was designed for a summary score [10].

In their overview of the characteristics of the evaluated instruments, Maratia et al. [1] concluded that there is no Spanish version available of the IBCSG QoL Core Form. In fact, the cross-cultural equivalence of the IBCSG indicators has been a crucial criterion during the development process. In a first initiative, the original English scale was translated into ten languages (German, Swedish, Slovenian, Italian, French, Spanish, Greek, Finish, Danish, and Norwegian) including Spanish. Forward and backward translations were performed by professional translators, followed by proofreading by IBCSG staff with mother tongue in the target language or professional translators. This process was completed by a testing with patients and adjusted with their feedback in order to assure not only linguistic but also conceptual equivalence of the questionnaire [11]. Patterns of scores over time were similar in the different language groups, and analyses of QoL changes over time in several IBCSG trials could be based upon pooled data after controlling for language/cultural group to obtain an overall treatment effect measure [11]. For more recent IBCSG trials (e.g., IBCSG SOFT and TEXT), the IBCSG QoL Core Form was translated in additional languages resulting in a total of over 20 languages by following international standard for the translation of patientreported outcomes [12].

Based on these misconceptions, we question the EMPRO rating presented by Maratia et al. [1] for several items and attributes, in particular:

\section{Involvement of target population}

In the early development phase of our indicators, cancer patients were involved by identifying 73 potential side effects resulting from cytotoxic chemotherapy [13]. Patients rated the seriousness of each side effect by using a predefined coding scheme. Only those side effects meeting a predefined cutoff ( 36 of 73 side effects) were retained for further analysis. Correlations between symptoms together with clinical judgment were used to group side effects into eight clusters resulting in the GLQ-8 [14] on which further development of the IBCSG QoL Core Form was based.

\section{Reliability: internal consistency}

Cronbach's alpha cannot be applied to determine the reliability of single-item measures. We assessed the test-retest reliability of the IBCSG QoL indicators by using 1- and 24-h intervals to ensure that the time interval was not too short so as to just test patients memory nor too long so that the patient's condition may have undergone real changes [14]. Correlations exceeded 0.65 for all indicators. We think that a correct rating here would be "not applicable."

\section{Content validity}

Maratia et al. strongly disagreed that the content validity of the IBCSG QoL Core Form was adequate. In our early publications on the development of the IBCSG approach, we presented content-related validity of the instrument for its intended use. The clinical relevance of global and specific LASA indicators has been confirmed in breast cancer trials both in the adjuvant and metastatic setting. These trials examined the impact of axillary clearance [15], adjuvant standard chemotherapy [16], dose-intensive chemotherapy [17], and endocrine therapy [18-20] on QoL, and the association between QoL and performance status [21], recurrence [16], tumor response [22], chemotherapy treatment benefit and toxicity [23], and survival duration $[24,25]$ in patients with metastatic disease.

\section{Prior hypothesis state}

For the individual QoL indicators, several validation steps have been described in different publications. For example, the mood and coping indicators were compared with standard measures of emotional well-being and psychosocial dysfunction $[6,7]$.

\section{Interpretability}

Maratia et al. [1] concluded that the scores for interpretability were lowest for the IBCSG QoL Core Form. The rationale for this low score is not clear based on the data presented. According to the rating of the EMPRO 
items and attributes summarized by Maratia et al. [1] in Table 3, the score for interpretability was derived from three different criteria. For two of these three criteria, the IBCSG QoL Core Form received the highest rating for agreement (i.e.,,++++ 4 , strongly agree). One criterion was rated with the option 'no information,' which applied when there was insufficient or inappropriate information. In comparison, the other scales reached similar ratings for two of three criteria and had also one criteria rated with "no information.' The total scores for interpretability should be similar among instruments with similar ratings.

In conclusion, the rating by Maratia et al. [1] regarding the IBCSG QoL Core Form is not correct and is in many respects misleading.

Funding There was no funding for this commentary.

\section{Compliance with ethical standards}

Conflict of interest All authors declare no conflict of interest.

Ethical approval In all studies referring to the IBCSG QoL Core Form cited in this commentary, procedures involving human participants were in accordance with the ethical standards of the institutional and/or national research committee and with the 1964 Helsinki Declaration and its later amendments or comparable ethical standards.

Informed consent Informed consent was obtained from all individual participants included in any of the studies referring to the IBCSG QoL Core Form cited in this commentary.

\section{References}

1. Maratia, S., Cedillo, S., \& Rejas, J. (2016). Assessing healthrelated quality of life in patients with breast cancer: A systematic and standardized comparison of available instruments using the EMPRO tool. Quality of Life Research, 25(10), 2467-2480. doi:10.1007/s11136-016-1284-8.

2. Bernhard, J., Hürny, C., Coates, A. S., Peterson, H. F., Castiglione-Gertsch, M., Gelber, R. D., et al. (1997). Quality of life assessment in patients receiving adjuvant therapy for breast cancer: The IBCSG approach. Annals of Oncology, 8(9), 825-835.

3. Valderas, J. M., Ferrer, M., Mendivil, J., Garin, O., Rajmil, L., Herdman, M., et al. (2008). Development of EMPRO: A tool for the standardized assessment of patient-reported outcome measures. Value Health, 11(4), 700-708. doi:10.1111/j.1524-4733. 2007.00309.x.

4. Bernhard, J., Peterson, H. F., Coates, A. S., Gusset, H., Isley, M., Hinkle, R., et al. (1998). Quality of life assessment in International Breast Cancer Study Group (IBCSG) trials: Practical issues and factors associated with missing data. Statistics in Medicine, 17(5-7), 587-601.

5. Butow, P., Coates, A., Dunn, S., Bernhard, J., \& Hurny, C. (1991). On the receiving end. IV: Validation of quality of life indicators. Annals of Oncology, 2(8), 597-603.

6. Bernhard, J., Sullivan, M., Hurny, C., Coates, A. S., \& Rudenstam, C. M. (2001). Clinical relevance of single item quality of life indicators in cancer clinical trials. British Journal of Cancer, 84(9), 1156-1165.

7. Hurny, C., Bernhard, J., Coates, A., Peterson, H. F., CastiglioneGertsch, M., Gelber, R. D., et al. (1996). Responsiveness of a single-item indicator versus a multi-item scale: assessment of emotional well-being in an international adjuvant breast cancer trial. Medical Care, 34(3), 234-248.

8. Hurny, C., Bernhard, J., Bacchi, M., van Wegberg, B., Tomamichel, M., Spek, U., et al. (1993). The Perceived Adjustment to Chronic Illness Scale (PACIS): A global indicator of coping for operable breast cancer patients in clinical trials. Swiss Group for Clinical Cancer Research (SAKK) and the International Breast Cancer Study Group (IBCSG). Supportive Care in Cancer, 1(4), 200-208.

9. Hürny, C., van Wegberg, B., Bacchi, M., Bernhard, J., Thürlimann, B., Ral, O., et al. (1998). Subjective health estimations (SHE) in patients with advanced breast cancer: an adapted utility concept for cancer clinical trials. British Journal of Cancer, 77, 985-991.

10. Michels, F. A. S., Latorre, R. D. O., \& Maciel, M. S. (2010). Validation, reliability, and comprehension of the IBCSG quality of life questionnaire specifi to Breast Cancer. Applied Cancer Research, 30(4), 348-352.

11. Hurny, C., Bernhard, J., Gelber, R. D., Coates, A., Castiglione, M., Isley, M., et al. (1992). Quality of life measures for patients receiving adjuvant therapy for breast cancer: An international trial. The International Breast Cancer Study Group. European Journal of Cancer, 28(1), 118-124.

12. Wild, D., Grove, A., Martin, M., Eremenco, S., McElroy, S., Verjee-Lorenz, A., et al. (2005). Principles of good practice for the translation and cultural adaptation process for patient-reported outcomes (PRO) measures: Report of the ISPOR task force for translation and cultural adaptation. Value Health, 8(2), 94-104. doi:10.1111/j.1524-4733.2005.04054.x.

13. Coates, A., Abraham, S., Kaye, S. B., Sowerbutts, T., Frewin, C., Fox, R. M., et al. (1983). On the receiving end-patient perception of the side-effects of cancer chemotherapy. European Journal of Cancer and Clinical Oncology, 19(2), 203-208.

14. Coates, A., Glasziou, P., \& McNeil, D. (1990). On the receiving end-III. Measurement of quality of life during cancer chemotherapy. Annals of Oncology, 1(3), 213-217.

15. International Breast Cancer Study G, Rudenstam, C. M., Zahrieh, D., Forbes, J. F., Crivellari, D., Holmberg, S. B., et al. (2006). Randomized trial comparing axillary clearance versus no axillary clearance in older patients with breast cancer: First results of International Breast Cancer Study Group Trial 10-93. Journal of Clinical Oncology, 24(3), 337-344. doi:10.1200/JCO.2005.01. 5784.

16. Hurny, C., Bernhard, J., Coates, A. S., Castiglione-Gertsch, M., Peterson, H. F., Gelber, R. D., et al. (1996). Impact of adjuvant therapy on quality of life in women with node-positive operable breast cancer. International Breast Cancer Study Group. Lancet, 347(9011), 1279-1284.

17. Bernhard, J., Zahrieh, D., Zhang, J. J., Martinelli, G., Basser, R., Hurny, C., et al. (2008). Quality of life and quality-adjusted survival (Q-TWiST) in patients receiving dose-intensive or standard dose chemotherapy for high-risk primary breast cancer. British Journal of Cancer, 98(1), 25-33. doi:10.1038/sj.bjc. 6604092.

18. Bernhard, J., Zahrieh, D., Castiglione-Gertsch, M., Hurny, C., Gelber, R. D., Forbes, J. F., et al. (2007). Adjuvant chemotherapy followed by goserelin compared with either modality alone: The impact on amenorrhea, hot flashes, and quality of life in premenopausal patients-the International Breast Cancer Study Group Trial VIII. Journal of Clinical Oncology, 25(3), 263-270. 
19. Bernhard, J., Luo, W., Ribi, K., Colleoni, M., Burstein, H. J., Tondini, C., et al. (2015). Patient-reported outcomes with adjuvant exemestane versus tamoxifen in premenopausal women with early breast cancer undergoing ovarian suppression (TEXT and SOFT): A combined analysis of two phase 3 randomised trials. Lancet Oncology, 16(7), 848-858. doi:10.1016/S14702045(15)00049-2.

20. Ribi, K., Luo, W., Bernhard, J., Francis, P. A., Burstein, H. J., Bellet, M., et al. (2016). Adjuvant tamoxifen plus ovarian function suppression versus tamoxifen-alone in premenopausal women with early breast cancer: patient-reported outcome in the SOFT trial. Journal of Clinical Oncology, 34(14), $1601-1610$.

21. Bernhard, J., Castiglione-Gertsch, M., Schmitz, S. F., Thurlimann, B., Cavalli, F., Morant, R., et al. (1999). Quality of life in postmenopausal patients with breast cancer after failure of tamoxifen: Formestane versus megestrol acetate as second-line hormonal treatment. Swiss group for clinical cancer research (SAKK). European Journal of Cancer, 35(6), 913-920.
22. Bernhard, J., Thurlimann, B., Schmitz, S. F., Castiglione-Gertsch, M., Cavalli, F., Morant, R., et al. (1999). Defining clinical benefit in postmenopausal patients with breast cancer under second-line endocrine treatment: does quality of life matter? Journal of Clinical Oncology, 17(6), 1672-1679.

23. Lee, C. K., Stockler, M. R., Coates, A. S., Gebski, V., Lord, S. J., \& Simes, R. J. (2010). Self-reported health-related quality of life is an independent predictor of chemotherapy treatment benefit and toxicity in women with advanced breast cancer. British Journal of Cancer, 102(9), 1341-1347.

24. Coates, A., Gebski, V., Signorini, D., Murray, P., McNeil, D., Byrne, M., et al. (1992). Prognostic value of quality-of-life scores during chemotherapy for advanced breast cancer. Australian New Zealand Breast Cancer Trials Group. Journal of Clinical Oncology, 10(12), 1833-1838.

25. Coates, A. S., Hürny, C., Peterson, H. F., Bernhard, J., Castiglione-Gertsch, M., Gelber, R. D., et al. (2000). Quality-of-life scores predict outcome in metastatic but not early breast cancer. Journal of Clinical Oncology, 18(22), 3768-3774. 